\title{
Mucocele labial em paciente pediátrico de 2 anos: relato de caso
}

\author{
Lip mucocele in a 2-year-old pediatric patient: case report \\ Mucocele labial en un paciente pediátrico de 2 años: relato de caso
}

Recebido: 22/12/2021 | Revisado: 29/12/2021 | Aceito: 30/12/2021 | Publicado: 31/12/2021

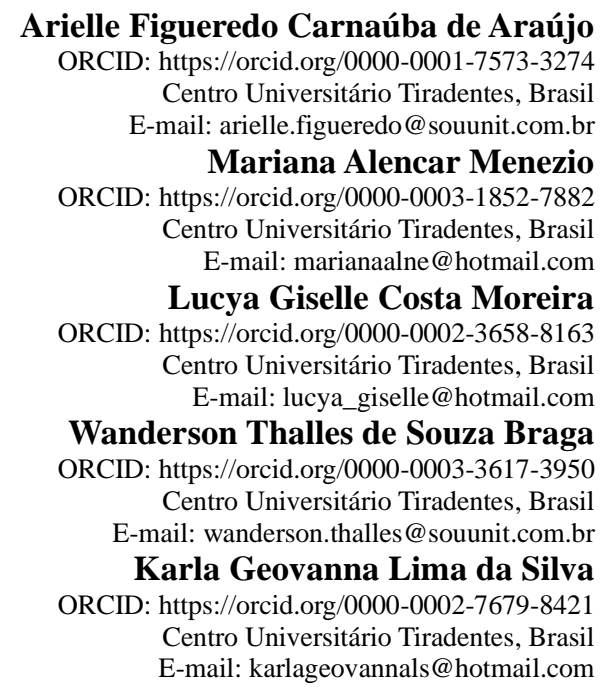

\section{Resumo}

Introdução: Mucocele é uma lesão benigna comum da mucosa oral e que se origina de glândulas salivares. O termo refere a uma cavidade contendo muco no seu interior, uma vez que o termo "cele" vem do grego "kele" que pode significar hérnia ou tumefação, sendo especialmente empregado para denominar cavidades vazias. Objetivo: $\mathrm{O}$ objetivo deste trabalho é relatar um caso clínico de mucocele labial em uma criança de 2 anos, removido por meio da excisão cirúrgica total da lesão. Relato de caso: Paciente do gênero feminino, leucoderma, compareceu a clínica de Odontopediatria do Centro Universitário Tiradentes - UNIT. A mãe relatou no momento da consulta que há uns 30 dias surgiu uma "bolinha" no lábio inferior da criança que doía quando a mesma se alimentava. Ao exame clínico foi observada uma lesão bolhosa, com limites nítidos, com aproximadamente $10 \mathrm{~mm}$ de diâmetro, de consistência fibrosa, móde base séssil, de coloração normocrômica, com características semelhantes de uma mucocele. Foi realizada a excisão cirúrgica da lesão e o material colhido foi fixado em formol a $10 \%$ e encaminhado para exame histopatológico. Posteriormente, foram recomendadas orientações pós-operatórias, bem como cuidados no local da lesão e prescrição de analgésico. O resultado do exame histopatológico confirmou o diagnóstico de cisto de retenção de muco (mucocele). Conclusão: A mucocele é a lesão mais comum em pacientes jovens e apresenta sinais patognomônicos característicos. Dentre as diversas técnicas terapêuticas o tratamento cirúrgico conservador ainda é a técnica mais utilizada, mostrando ser uma conduta eficaz, simples e de bom prognóstico.

Palavras-chave: Mucocele; Odontopediatria; Cirurgia.

\begin{abstract}
Introduction: Mucocele is a common benign lesion of the oral mucosa that originates from salivary glands. The term refers to a cavity containing mucus in its interior, since the term "cele" comes from the Greek "kele" which can mean hernia or swelling, being especially used to name empty cavities. Objective: The objective of this work is to report a clinical case of labral mucocele in a 2-year-old child, removed by total surgical excision of the lesion. Case report: Female patient, leucoderma, attended the Pediatric Dentistry clinic of Centro Universitário Tiradentes - UNIT. The mother reported at the time of the consultation that, about 30 days ago, a "ball" appeared in the child's lower lip, which hurt when she was eating. On clinical examination, a bullous lesion was observed, with clear limits, approximately $10 \mathrm{~mm}$ in diameter, fibrous consistency, sessile-based mould, normochromic coloration, with characteristics similar to those of a mucocele. Surgical excision of the lesion was performed and the collected material was fixed in $10 \%$ formalin and sent for histopathological examination. Afterwards, postoperative guidelines were recommended, as well as care at the site of the injury and prescription of analgesics. The result of the histopathological examination confirmed the diagnosis of a mucus retention cyst (mucocele). Conclusion: Mucocele is the most common lesion in young patients and presents characteristic pathognomonic signs. Among the various
\end{abstract}


therapeutic techniques, conservative surgical treatment is still the most used technique, proving to be an effective, simple approach with a good prognosis.

Keywords: Mucocele; Pediatric dentistry; Surgery.

\begin{abstract}
Resumen
Introducción: El mucocele es una lesión benigna común de la mucosa oral que se origina en las glándulas salivales. El término se refiere a una cavidad que contiene moco en su interior, ya que el término "cele" proviene del griego "kele" que puede significar hernia o hinchazón, siendo especialmente nominal para nombrar cavidades vacías. Objetivo: El objetivo de este trabajo es informar caso clínico de mucocele del labrum en un niño de 2 años, que remitió mediante exéresis quirúrgica total de la lesión. Relato del caso: Paciente de sexo femenino, de raza blanca, que acudió a la consulta de Odontopediatría del Centro Universitário Tiradentes - UNIT/AL. La madre informó en el momento de la consulta que, hace unos 30 días, apareció una "bola" en el labio inferior de la niña, que le dolía cuando comía. Al examen clínico se observó una lesión ampollosa, de límites claros, de aproximadamente $10 \mathrm{~mm}$ de diámetro, de consistencia fibrosa, de base más sésil, de color normocrómico, con características similares a un mucocele. Se realizó escisión quirúrgica de la lesión y el material recolectado se corrigió en formalina al $10 \%$ y se envió para examen histopatológico. Posteriormente se recomendó pautas postoperatorias, así como cuidados en el sitio de la lesión y prescripción de analgésicos. El resultado del examen histopatológico confirmó el diagnóstico de un quiste de retención de moco (mucocele). Conclusión: El mucocele es la lesión más común en pacientes jóvenes y presenta signos patognomónicos característicos. Entre las diversas técnicas terapéuticas, el tratamiento quirúrgico conservador sigue siendo el más utilizado, demostrando ser un abordaje eficaz, sencillo y con buen pronóstico.
\end{abstract}

Palabras clave: Mucocele; Odontología pediatrica; Cirugía.

\title{
1. Introdução
}

A mucocele caracteriza-se por ser uma lesão benigna da cavidade bucal que se desenvolve através do extravasamento ou retenção de muco (Santos et al., 2013; Bezerra et al., 2016; Magalhães et al., 2020). É uma lesão que envolve glândulas e ductos salivares maiores ou menores (Magalhães et al., 2020), sendo sua patogenia relacionada principalmente ao trauma sobre o ducto salivar excretor ou a obstrução do mesmo (Santos et al., 2013; Rocha et al., 2013). Sua taxa de incidência é geralmente alta sendo a $17^{\circ}$ lesão mais comum da cavidade bucal, acometendo cerca de $0,08 \%$ das lesões orais no Brasil (Bezerra et al., 2016).

As características clínicas, bem como sua localização podem surgir em qualquer região da mucosa bucal que possua glândula salivar devido essa lesão representar 70\% das lesões císticas e pseudocísticas (Stuani et al., 2010; Bezerra et al., 2016), no entanto, aproximadamente 81,9\% acometem o lábio inferior sendo o sítio de maior predileção (Santos et al., 2013). Apresenta-se como uma elevação superficial, macia à palpação, assintomática, de coloração igual à mucosa adjacente ou levemente azulada e de tamanho variado, podendo em alguns casos ultrapassar 10mm de diâmetro (Danelon et al., 2013; Rocha et al., 2013; Santos et al., 2013; Bezerra et al., 2016; Magalhães et al., 2020). As recorrências dessas lesões são frequentes, embora possam romper espontaneamente e não haver recidiva (Danelon et al., 2013).

Histologicamente pode-se apresentar de duas formas: fenômeno de retenção ou extravasamento de muco (Bezerra et al., 2016). O fenômeno de retenção de muco caracteriza-se por possuir revestimento epitelial proveniente do ducto salivar obstruído (Danelon et al., 2013; Magalhães et al., 2020), geralmente está associado as glândulas salivares maiores e fatores etiológicos (Bezerra et al., 2016). Nesse ínterim, os fenômenos de extravasamento de muco não possuem revestimento epitelial, dando origem de fato a um pseudocisto, esse tipo de mucocele ocorre principalmente nas glândulas salivares menores (Stuani et al., 2010; Bezerra et al., 2016).

O diagnóstico é baseado no quadro clínico característico no qual deve ser confirmado pela análise histopatológica (Nascimento et al., 2014; Albuquerque et al., 2015). A literatura aborda várias formas de tratamento como a microcirurgia, marsupialização, micromarsupialização e a aplicação do laser de argônio, no entanto, a excisão cirúrgica ainda é o tratamento mais utilizado por evitar recorrências (Stuani et al., 2010). O objetivo desse trabalho é relatar um caso clínico de mucocele labial em uma criança de 2 anos e sua conduta terapêutica. 


\section{Metodologia}

Foram realizadas todas as etapas de anamnese, exame físico e de imagem, em seguida foi explicada a abordagem que seria realizada para a responsável, salientando a necessidade do termo de consentimento livre e esclarecido (TCLE). O trabalho trata-se de um estudo analítico descritivo (Pereira et al., 2018), realizado na clínica odontológica do Centro Universitário Tiradentes - UNIT/AL.

\section{Relato de Caso}

Paciente de 2 anos, sexo feminino, leucoderma, foi encaminhada à clínica de odontopediatria do Centro Universitário Tiradentes - UNIT/AL para consulta de urgência. A mãe relatou ter observado que há 30 dias surgiu uma "bolinha" no lábio inferior de sua filha e quando a mesma se alimentava apresentava dor ou incômodo. Ao exame clínico, observou-se uma lesão bolhosa no lábio inferior esquerdo, séssil, nodular, consistência fibrosa, com limites nítidos, medindo aproximadamente $10 \mathrm{~mm}$ de diâmetro, de coloração normocrômica e superfície lisa (Figura 1A). Diante das características clínicas encontradas, o diagnóstico clínico provável da lesão foi determinado como mucocele labial. Mediante ao quadro clínico característico a conduta terapêutica adotada foi à remoção cirúrgica da lesão para exame histopatológico. Após os cuidados pré-operatórios, foi realizada a anestesia local infiltrativa na mucosa (Figura 1B), ao redor da lesão com o intuito de não deformá-la. Posteriormente, a lesão foi apreendida com a pinça Allis e a incisão feita com a lâmina de bisturi número 15 realizada cuidadosamente para que não houvesse perfuração (Figura 1C).

Figura 1: (A) Aspecto clínico inicial da lesão na mucosa labial inferior. (B) Técnica anestésica local. (C) Apreensão com pinça Allis e incisão com lâmina de bisturi n ${ }^{\circ} 15$.
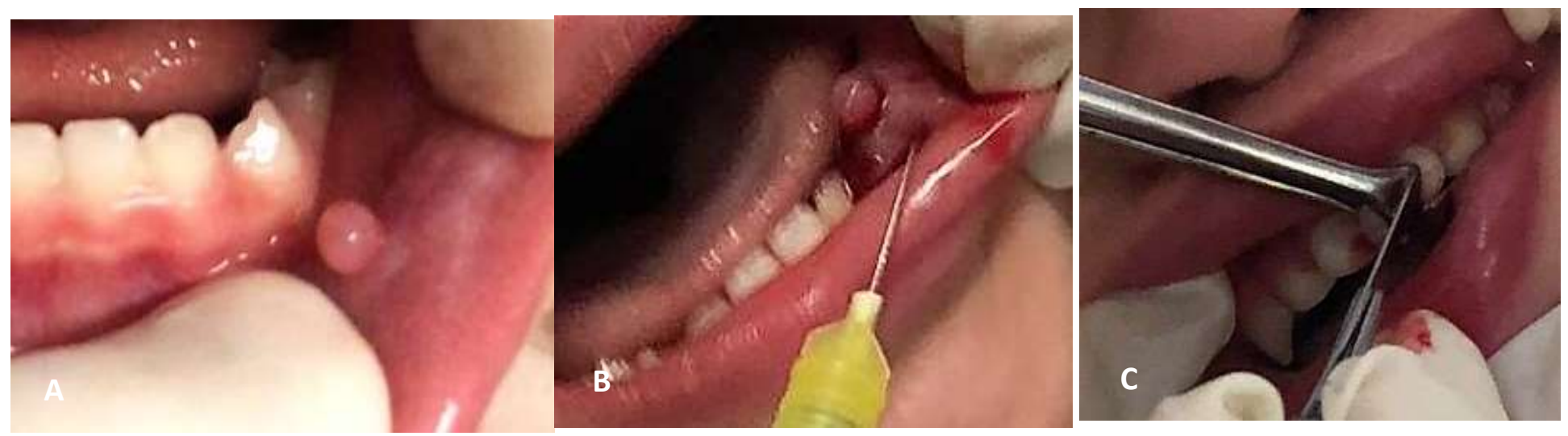

Fonte: Acervo pessoal dos autores.

O tecido foi divulsionado e a excisão cirúrgica da lesão executada (Figura 2A), o material colhido foi fixado em formol a $10 \%$ e encaminhado para o exame histopatológico (Figura 2B). A sutura foi realizada e o paciente retornou após 7 dias para remoção dos pontos cirúrgicos. Após a cirurgia, a mãe foi orientada sobre os cuidados pós-operatórios e sobre as precauções com a ferida cirúrgica. A prescrição de analgésico foi necessária nas primeiras 24 horas. A cicatrização da ferida cirúrgica é observada e o prognóstico é favorável (Figura 2C). 
Figura 2: (A) Exposição após incisão. (B) Mucocele removida imersa em formol 10\%. (C) Aspecto final e processo de cicatrização da ferida cirúrgica.
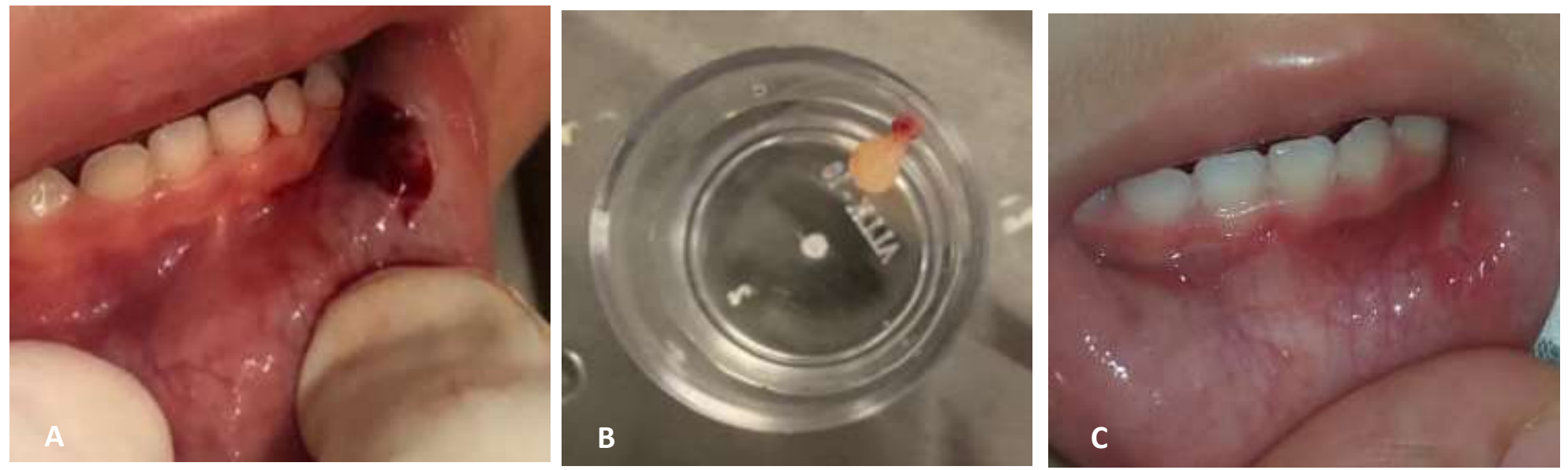

Fonte: Acervo pessoal dos autores.

O diagnóstico definitivo da peça cirúrgica confirmou a hipótese diagnóstico de mucocele, uma vez que o resultado da análise anatomopatológica revelou ' "cisto de retenção de muco'. O paciente foi mantido em acompanhamento e não apresentou recidiva após 4 meses da excisão cirúrgica da lesão.

\section{Discussão}

A mucocele é uma das lesões benignas que mais afeta a cavidade bucal de crianças (Stuani et al., 2010; Santos et al., 2013; Bezerra et al., 2016; Magalhães et al., 2020), seu desenvolvimento pode ser devido a uma obliteração do ducto excretor da glândula salivar o que resulta no retorno do muco ou através de traumatismos no ducto salivar que gera uma evasão de muco para os tecidos adjacentes (Magalhães et al., 2020; Mariano et al., 2004; Freitas et al., 2012). O caso clínico apresentado reforça os demais artigos que as mucoceles são lesões orais comuns em pacientes jovens, geralmente decorrentes de traumas sob os ductos salivas devido a hábitos deletérios.

A literatura aponta que as mucoceles podem acometer diversas regiões da cavidade bucal como o lábio, mucosa jugal, bochecha, palato, língua e assoalho bucal (Mariano et al., 2004; Chi et al., 2011; Manfro et al., 2011; Freitas et al., 2012), todavia cerca de aproximadamente 44 a 79\% dos casos acomete o lábio inferior (Santos et al., 2013), como no caso clínico apresentado. Os autores não chegam a um consenso quanto à predileção de gênero e idade (Danelon et al., 2013; Santos et al., 2013; Rocha et al., 2013), no entanto, Neville et al. (2014) aponta uma maior prevalência no gênero feminino o que corrobora com o caso clínico relatado.

Os autores apontam que a apresentação clínica da mucocele oral pode variar, no entanto seus aspectos clínicos são patognomônicos como: lesão nodular, assintomática, superfície lisa e com uma variedade de cores (Mariano et al., 2004; Stuani et al., 2010; Danelon et al., 2013; Rocha et al., 2013; Santos et al., 2013; Bezerra et al., 2016; Magalhães et al., 2020). Esses achados podem ser evidenciados no presente caso clínico, diferindo apenas na sintomatologia dolorosa.

Diferentes técnicas terapêuticas são propostas na literatura, a micromarsupialização consiste na epitelização ao redor de um fio de sutura resultando no desaparecimento da lesão (Stuani et al., 2010; Castillo et al., 2011; Rocha et al., 2013; Sukhtankar et al., 2013), sendo contra-indicada em lesões localizadas no palato e na mucosa jugal (Stuani et al., 2010). Na marsupialização é realizada a remoção superficial da lesão e a sutura do ducto salivar com o epitélio bucal para que a 
drenagem possa ser mantida, sendo sua desvantagem a alta taxa de recidiva (Alves et al., 2010; Stuani et al., 2010; Martínez et al., 2014). A criocirurgia é bastante aceita em pacientes pediátricos por não precisar de anestesia durante o procedimento, uma vez que a técnica consiste em apenas aplicar um agente criogênico sobre a lesão (Stuani et al., 2010; Freitas et al., 2012; Sukhtankar et al., 2013), mas durante as primeiras aplicações a lesão tende a recidivas (Stuani et al., 2010). A aplicação do laser de argônio utiliza-se um raio laser que resultará na necrose da lesão, sendo seu alto custo uma desvantagem (Stuani et al., 2010; Sukhtankaret al., 2013; Paglia et al., 2015). Devido essas técnicas apresentar aumento de recidivas dos casos, a excisão cirúrgica tem sido a principal opção terapêutica (Mariano et al., 2004; Stuani et al., 2010; Castillo et al., 2011; Santos et al., 2013; Corteleti et al., 2015; Magalhães et al., 2020). No caso clínico, após a exérese da lesão o paciente apresentou resultados satisfatórios sem recidivas ou novas lesões induzindo ao sucesso do tratamento e corroborando com os demais autores.

\section{Considerações Finais}

A mucocele é a lesão mais comum em pacientes jovens e apresenta sinais patognomônicos característicos. Dentre as diversas técnicas terapêuticas o tratamento cirúrgico conservador ainda é a técnica mais utilizada, mostrando ser uma conduta eficaz, simples e de bom prognóstico.

\section{Referências}

Albuquerque, A. C. L., Rodrigues, F. G., Soares, M. S. M., \& Silva, D. F. (2015). Diagnóstico e tratamento de mucocele labial: relato de caso. Revista Saúde \& Ciência Online, 4(1), 25-31.

Alves, L. A., Di Nicoló. R., Ramos, C. J., Shintome, L. \& Barbosa, C. S. (2010). Mucocele de retenção em lábio inferior associada ao uso inadequado de chupeta. Dermatol J. 16 (7).

Bezerra, T. M., Bárbara, V. M., Águida, C. G., Henriques, M. V. C., Cassiano, F. W. N., Márcia, C. C. M. (2016). Levantamento epidemiológico de fenômeno de extravasamento de muco de um centro de referência em patologia oral por um período de 43 anos. Braz. j. otorhinolaryngol, 82(5), $536-542$.

Castillo, H. A. A. (2011). Uso de OK-432 (Picibanil) como alternativa no quirúrgica para el manejo de ránulas y mucoceles. Revista de la Asociación Dental Mexicana, 68(5), 215-221.

Chi, A. C., Lambert III, P. R., Richardson, M. S., \& Neville, B. W. (2011). Oral mucoceles: a clinicopathologic review of 1,824 cases, including unusual variants. Journal of Oral and Maxillofacial Surgery, 69(4), 1086-1093.

Corteleti, J. F., Ota, C. M., Hesse, D., Novaes, T. F., Raggio, D. P., \& Imparato, J. C. P. (2015). Remoção cirúrgica de fibroma lingual e gengival em crianças. Revista da Associacao Paulista de Cirurgioes Dentistas, 69(1), 30-35.

Danelon, M., Lodi, C. S., Favretto, C. O., Crivelini, M. M., Cunha, R. F., \& Delbem, A. C. B. (2013). Diagnóstico e tratamento de mucocele em odontopediatria: relato de caso. Archives of Health Investigation, 2(5).

Freitas, M. D. C. A., Falcão, M. M. L., Ramos, M. E. S. P., Batista, T. S., Santos, 1. D. P. D. S., \& Braga, T. R. (2012). Terapêutica interdisciplinar na mucocele oral: relato de caso. Revista Uningá, 31(1).

Magalhaes, L. S., Calazans, M. N. B., Cota, A. L. S., Nemezio, M. A., Junior, M. A. B. (2020). Diagnosis and treatment of mucocele in a pediatric patient: case report. RGO, Rev. Gaúch. Odontol., 68(2).

Manfro, A. R. G., Manfro, R., \& Bortoluzzi, M. C. (2010). Mucocele em lábio inferior-Relato de caso clínico. Unoesc \& Ciência-ACBS, 1(2), $135-140$.

Mariano, C. V., Pikunas, C., Miguel, C. M., Nakamura, L. K., Imparato, J. C. P., Pinheiro, S. L. (2004). Mucocele. Rev Ciênc Méd, 13(1), 77-83.

Martínez, A., Arcila, V. G., Carmona, Z. (2014). Resección quirúrgica de mucocele utilizando relleno siliconado. Salud Uninorte, 30(3), 498-504.

Nascimento, J. S., Azevedo, R. S., Barros, E. M. V. B., Takahama, J. A. (2014). Mucoceles da cavidade oral: análise das características histopatológicas de 42 casos. Rev Odontol Bras Central, 23(66), 162-165.

Neville, B. W., Damm, D., Allen, C. M., Bouquot, J. E. (2004). Patologia oral e maxillofacial. Guanabara Koogan, (2).

Paglia, M., Crippa, R., Ferrante, F., \& Angiero, F. (2015). Mucocele of the minor salivary glands in an infant: Treatment with diode laser. Eur J Paediatr Dent, 16(2), 139-42.

Pereira, A. S., Shitsuka, D. M., Parreira, F. J., \& Shitsuka, R. (2018). Metodologia da pesquisa científica.

Rocha, A. L., Basílio, J. M. B. S., Amaral, M. B. F., \& Mesquita, R. A. (2013). Tratamento da mucocele com a técnica da micromarsupialização modificada. Revita da Associação Paulista de Cirurgiões Dentistas, 67(4), 268-271 
Research, Society and Development, v. 10, n. 17, e256101725057, 2021

(CC BY 4.0) | ISSN 2525-3409 | DOI: http://dx.doi.org/10.33448/rsd-v10i17.25057

Santos, F. M., Corrêa, F. N. P., \& Corrêa, M. S. N. P. (2013). Mucocele em lábio inferior de adolescente: relato de caso. Revista da Associação Paulista de Cirurgiões Dentistas; 67(3), 230-233.

Stuani, A. S., Stuani, A. S., de Paula, F. W. G., Silva, M. B. S. S., Valério, R. A., \& Mussolino, A. (2010). Mucoceles: lesões frequentes na cavidade bucal de crianças Mucoceles: frequent lesions in children's mouth. Pediatria (São Paulo), 32(4), 288-92.

Sukhtankar, L. V., Mahajan, B., \& Agarwal, P. (2013). Treatment of lower lip mucocele with diode laser-A novel approach. Ann Dent Res, 2(Suppl 1), 102-8. 